This document is the accepted manuscript version of the following article:

Anthis, A. H. C., Matter, M. T., Keevend, K., Gerken, L., Scheibler, S., Doswald, S., ... Herrmann, I. K. (2019). Tailoring the Colloidal Stability, Magnetic Separability and Cytocompatibility of High-Capacity Magnetic Anion Exchangers. ACS Applied Materials and Interfaces. https://doi.org/10.1021/acsami .9b16619

\title{
Tailoring the Colloidal Stability, Magnetic Separability and Cytocom- patibility of High-Capacity Magnetic Anion Exchangers
}

\author{
Alexandre H.C. Anthis ${ }^{1,2}$, Martin T. Matter ${ }^{1,2,3}$, Kerda Keevend ${ }^{1,2}$, Lukas R.H. Gerken ${ }^{1,2}$, Subas Scheibler ${ }^{1,2,4}$, \\ Simon Doswald ${ }^{5}$ Alexander Gogos ${ }^{1}$ and Inge K. Herrmann ${ }^{1,2 *}$
}

\begin{abstract}
${ }^{1}$ Laboratory for Particles Biology Interactions, Department Materials Meet Life, Swiss Federal Laboratories for Materials Science and Technology (Empa), Lerchenfeldstrasse 5, CH-9014 St. Gallen, Switzerland.

${ }^{2}$ Nanoparticle Systems Engineering Laboratory, Institute of Process Engineering, Department of Mechanical and Process Engineering, ETH Zurich, Sonneggstrasse 3, CH-8092 Zurich, Switzerland ${ }^{3}$ Particle Technology Laboratory, Institute of Process Engineering, Department of Mechanical and Process Engineering, ETH Zurich, Sonneggstrasse 3, CH-8092 Zurich, Switzerland ${ }^{4}$ Laboratory for Nanoscale Materials Science, Department Materials Meet Life, Swiss Federal Laboratories for Materials Science and Technology (Empa), Überlandstrasse 129, CH-8600 Dübendorf, Switzerland.

${ }^{5}$ Functional Materials Laboratory, Institute for Chemical and Bioengineering, Department of Chemistry and Applied Biosciences, ETH Zurich, Vladimir-Prelog-Weg 1, CH-8093 Zurich, Switzerland.
\end{abstract}

*inge.herrmann@empa.ch, ingeh@ethz.ch, +41 (0)58 7657153 


\begin{abstract}
Extracorporeal blood purification has been applied to artificially support kidney or liver function. However, convection and diffusion based blood purification systems have limited removal rates for high molecular weight and hydrophobic molecules. This limitation is due to the finite volume of infusion and limited membrane permeability, respectively. Adsorption provides an attractive alternative for the removal of higher molecular weight compounds. The use of adsorption resins containing ion exchanging groups to capture specific molecules has become well-established. Instead of stationary adsorption resins, however, ion exchanging polymers may be immobilized on magnetic particles and serve as freely diffusing, mobile, high capacity solid phase of ion exchange chromatography. While small beads with high surface area are attractive in terms of mass transfer and binding, unifying high capturing capacity with rapid and quantitative bead recovery remains an issue. Therefore, most of the current magnetic ion exchangers are based on micron-sized beads or require long times to separate. In addition to unfavourable magnetic recovery rates, the usually poor cytocompatibility limits their applicability in biomedicine.

Here, we report on the synthesis and performance of polycationic polymer coated magnetic nanoflowers (MNF) for highly efficacious anion capturing. We demonstrate accurate control over the polymer content and composition on the beads, and show its direct influence on colloidal stability, capturing capacity and magnetic separability. We present the removal of clinically relevant targets by capturing bilirubin with capacities twofold higher than previous work as well as quantitative heparin removal. Additionally, we illustrate how copolymerization of poly(2-dimethylaminoethyl methacrylate) (PDMAEMA) with poly(ethylene glycol) methyl ether methacrylate (PEGMEMA) leads to improved cytocompatibility of the polymer-coated MNF capturing agents while retaining high capturing capacities. Taken together, we present a nanoparticle/polymer material, which upon future in vivo validation, unifies high binding capacities and magnetic separability for rapid toxin capturing and hence fulfils key requirements of clinical utility.
\end{abstract}

Keywords: resin, nanoparticles, polymer, blood purification, ion exchange 


\section{INTRODUCTION}

Despite advances in intensive care, mortality rates in patients suffering from liver failure remain disturbingly high. ${ }^{1,2}$ The clinical presentation of liver failure typically includes accumulation of toxic metabolites that are no longer adequately cleared. Liver failure often involves the rapid deterioration of the mental status and progression to fatal multi-organ failure in up to half of the cases. ${ }^{1,3}$ In order to stabilize the patients while they await transplantation, or to facilitate native liver regeneration, artificial liver support (ALS) technologies have been developed to remove accumulating endogenous toxins (e.g. bilirubin) as well as unwanted drugs (e.g. heparin). ${ }^{4}$

Since filter and stationary adsorbent based extracorporeal detoxification systems are oftentimes mass-transfer limited, ${ }^{5}$ magnetic nanoparticle-based blood purification offers an attractive alternative for the efficient cleansing of body fluids. ${ }^{6-8}$ Freely accessible nanoparticles uniformly dispersed in the fluid offer an increased surface area for binding and smaller diffusion distances. ${ }^{5,9}$ In extracorporeal blood purification, the residence time of blood in the extracorporeal circuit is typically limited to a few minutes in order to ensure sufficient throughput. Consequently, sorbent/body-fluid contact times are short, and rapid binding as well as fast magnetic recovery are essential. While nanoparticulate beads are highly beneficial in terms of diffusion lengths as well as binding capacity (per sorbent mass), small particles are challenging to remove because of weak net magnetic forces due to the low particle mass. ${ }^{9}$ In intoxications, the removal of specific targets with specific capturing agents is desirable and has been demonstrated for a diversity of substances. ${ }^{10,11}$ However, in conditions such as systemic inflammation or liver failure, where there are multiple (unidentified) disease causing factors circulating in the blood, broadband capturing agents are more appealing. ${ }^{12}$ Both charcoal as well as resin-based cartridges, including anion exchange resins, ${ }^{13,14}$ have been successfully used in preclinical and clinical settings for the treatment of liver failure. ${ }^{15}$ Recently, the successful removal of bilirubin, a toxic degradation product of haemoglobin, has been achieved by magnetic particle sorbent systems based on poly(2-hydroxyethyl methacrylate) immobilised albumin, ${ }^{16}$ nitrogen-doped porous carbon, ${ }^{17}$ or magnetic multi-walled carbon nanotubes. ${ }^{18}$ These magnetically separable sorbents showed maximal bilirubin binding capacities of $\sim 260 \mathrm{mg}$ per g. However, the existing magnetic sorbents oftentimes suffer from weak saturation magnetizations (typically $<10$ emu/g) and slow binding kinetics (hours). These drawbacks decisively limit their applicability in continuous extracorporeal body fluid purification, where residence times are constrained to minutes. Alternatively, the use of polymer-brush functionalized magnetic nanoparticles is especially promising due to high binding capacities 
of cationic polymer brushes. ${ }^{19}$ For the immobilization of polymer brushes on surfaces, various strategies have been reported, including the "grafting-onto" and "grafting-from" methods. ${ }^{20}$ While "grafting-from" methods generally allow high density polymer brush architectures, the functionalization of magnetic nanoparticles with densely packed polymer has been shown to greatly compromise their separability due to steric and electrostatic stabilization. Even ferromagnetic nanoparticles with saturation magnetizations as high as $150 \mathrm{emu} / \mathrm{g}$ have been stabilized by cationic polymer brush grafting to the extent that they were no longer magnetically separable. ${ }^{21,22}$ Thus, excellent control over the amount of polymer added to the particle surface is needed in order to achieve toxin binding capacity while still allowing complete bead recovery.

Here, we report the synthesis and functionalization of magnetic iron oxide nanoflowers (MNF) with polycationic polymer brushes (Scheme 1) for highly efficacious anion capturing from body fluids. We demonstrate rapid ( $<3$ minutes) and efficient capturing of two clinically relevant substances, bilirubin and heparin. Additionally, we show that the cyto- and hemolytic properties of the polycationic polymer brushes can be attenuated by employing co-polymers that retain high anion capturing capacities.

\section{RESULTS AND DISCUSSION}

Magnetic iron oxide nanoflowers (MNF) were prepared via a surfactant free solvothermal method. ${ }^{23}$ The particle size distribution $\left(\mathrm{d}_{\text {TEM }}\right)$ measurements based on the transmission electron micrographs (TEM) (Figure 1a) indicate a uniform size with a mean diameter of $210 \pm 70 \mathrm{~nm}$ (Figure 1b). X-Ray diffraction (XRD) patterns show sharp diffraction peaks indicating high crystallinity (Figure 1c). Peak intensities and positions accurately correspond to that of magnetite. The crystalline structure of the particles was also confirmed using high resolution transmission electron micrographs (HR-TEM) and selected area electron diffraction (SAED) (see ESI Figure $\mathrm{S} 1)$. The MNF are composed of small primary crystallites with average diameters $\left(\mathrm{d}_{\mathrm{XRD}}\right)$ of $25 \pm 2 \mathrm{~nm}$ as estimated by the Scherrer equation. The saturation magnetization determined by vibrating sample magnetometry (VSM, Figure 1d) is $62 \mathrm{emu} / \mathrm{g}$ (equivalent to $\sim 160 \mathrm{emu} / \mathrm{g} \mathrm{Fe}$ ), which is in line with previous reports. ${ }^{23}$ Following physicochemical characterization, the MNF were functionalized with a thin layer (10 $\mathrm{wt} \%)$ of polydopamine (PDA)-based polymerization initiator (PDA-Br, Figure 1e) before being subjected to a surface initiated atom transfer radical polymerization (SI-ATRP). The amount of PDA deposited on the MNF surface can be well controlled as it is directly proportional to the reaction time (Figure 1f). Polycationic polymer brushes based on 
dimethylaminoethyl acrylate (DMAEMA) were then grown from the surface of MNF-PDA-Br (with $10 \mathrm{wt} \%$ loading) by SI-ATRP ${ }^{24}$ and subsequently quaternized in order to improve colloidal stability and introduce functional units for toxin capturing. The different surface functionalization steps were characterized by a combination of Fourier transform infrared spectroscopy (FTIR) and thermogravimetric analysis (TGA). Following the first reaction, FTIR spectra show bands at $1484 \mathrm{~cm}^{-1}$, and $1253 \mathrm{~cm}^{-1}$ characteristic for polydopamine (PDA) ${ }^{25}$ (Figure 1e). The amount of initiator was then quantified by elemental analysis, after thoroughly washing the nanoparticles in a series of different solvents, as $156 \mu \mathrm{mol} / \mathrm{g}$. The subsequent SI-ATRP polymerization of DMAEMA was confirmed by FTIR with indicative bands of PDMAEMA at $1716 \mathrm{~cm}^{-1}(\mathrm{C}=\mathrm{O}), 2763 \mathrm{~cm}^{-1}, 2939$ $\mathrm{cm}^{-1}(\mathrm{C}-\mathrm{H})$ and $1142 \mathrm{~cm}^{-1}(\mathrm{C}-\mathrm{N}) \cdot{ }^{24,26}$ The disappearance of peaks at 2763 and $2816 \mathrm{~cm}^{-1}(\mathrm{C}-\mathrm{H}$ bonds of the $\left.\mathrm{N}\left(\mathrm{CH}_{3}\right)_{2}\right)$ from the MNF-PDMAEMA particles and the appearance of new peaks at 1618 and $945 \mathrm{~cm}^{-1}$ are associated with the charged quaternized amine on the spectrum of the quaternized PDMAEMA indicating successful quaternization of the amines ${ }^{16,17}$ (Figure 1e). Following the "grafting-from" polymerization on the nanoparticle surface, the polymer loading was determined as a function of reaction time by thermogravimetric analysis (TGA) and elemental analysis (CHN). The relationship between the polymerization time and the polymer mass grafted from the particle surface was linear for the first 18 hours reaching a plateau value afterwards (Figure 1g). Based on the concentration of initiator sites, the added mass, and the assumption of 100\% initiation efficiency, molecular weights of the polymers were estimated as $400 \mathrm{~g} / \mathrm{mol}$ (MNF-PDMAEMA(S)), $700 \mathrm{~g} / \mathrm{mol}$ (MNF-PDMAEMA(M)), and $2300 \mathrm{~g} / \mathrm{mol}$ (MNF-PDMAEMA(L)) for reaction times of 1.5, 4 and 24 hours, respectively. Where not stated otherwise, MNF-PDMAEMA with the highest loading (MNF-PDMAEMA(L)) were used for further experiments. Based on TGA (see ESI, Figure S2), the extent of quaternization was estimated as $\sim 30 \%$ for the full length MNF-qPDMAEMA(L). Steric hindrance has previously been identified to have a key impact on quaternization efficiency. ${ }^{27}$ Quaternization yields for MNF-qPDMAEMA(S) and MNFqPDMAEMA(M) were $100 \%$ and $60 \%$, respectively, and in the range of previously reported quaternization yields for nanoparticulate systems. ${ }^{28}$ In addition to MNF functionalized with quaternized PDMAEMA (MNFqPDMAEMA), DMAEMA was co-polymerized with either poly(ethylene glycol) methyl ether methacrylate (PEGMEMA) or poly(methyl methacrylate) (PMMA), in order to obtain the copolymer-functionalized beads MNF-qPDMAEMA-co-PEGMEMA and MNF-qPDMAEMA-co-PMMA, respectively. The polymer loadings for the three polymer-MNF systems were comparable (see ESI, Figure S3 and Table S1). The monomer number 
ratio DMAEMA/PEGMEMA was found to be $\sim 2.95$ based on the elemental analysis (see ESI, Table S1), which is in good agreement with the theoretical ratio of 3 , calculated from the as added molar amounts of the two monomers, assuming perfect mixing. The saturation magnetization of the functionalized MNF remained $\sim 160$ $\mathrm{emu} / \mathrm{g} \mathrm{Fe}$ indicating no further oxidation of the core by surface functionalization.

The hydrodynamic size $\left(\mathrm{d}_{\mathrm{H}}\right)$ of the functionalized MNF determined by dynamic light scattering (DLS) shows a significant increase, which linearly correlates $\left(\mathrm{R}^{2}=0.99\right)$ with the polymer loading (Figure 2a). Zeta potential measurements confirm successful functionalization and quaternization of the pDMAEMA giving average values of $-34 \mathrm{mV}$ for bare MNF, $+25 \mathrm{mV}$ for MNF-PDMAEMA and $+39 \mathrm{mV}$ for MNF-qPDMAEMA. MNFqPDMAEMA-co-PEGMEMA show a slightly lower positive charge of $+38 \mathrm{mV}$ (Figure $2 \mathbf{b}$ ). To characterize the colloidal stability of the functionalized MNF, sedimentation over time was investigated by UV/Vis spectroscopy in phosphate buffered saline (PBS) with an ionic strength of $150 \mathrm{mM}$ (Figure 2c, d). Colloidal stability is highly dependent on the polymer loading and the ionic strength, and is significantly reduced in PBS as compared to water (see also ESI, Figure S4). Colloidal stability increases as a function of the qPDMAEMA polymer loading and is slightly reduced by the introduction of PEGMEMA units. Colloidal stability of the MNF was significantly improved from minutes (for the bare MNF) to 2 hours by the full-length qPDMAEMA coating, without detectable sedimentation. The improved colloidal stability was also confirmed by recording transmission electron micrographs of particles recovered from suspensions, which show no indication of particle coagulation (Figure 2e). Derjaguin-Landau-Verwey-Overbeek (DLVO) calculations show that the MNF were stabilized mostly by steric repulsion, which exceeded the electrostatic contribution (see ESI, Figure S5), in agreement with previous work on polymer-functionalized carbon-coated cobalt nanoparticles. ${ }^{16}$ While a fraction of the particles $(\sim 30 \%)$ remained in suspension for $\geq 24$ hours, subsequent magnetic separation allowed instant quantitative bead recovery (see ESI, Figure S4).

Colloidal stability is of high importance for efficient interaction and binding, however, it also counteracts efficient magnetic separation. Therefore, magnetic separation efficiency was assessed as a function of dispersant composition and separation time by UV absorbance (see ESI, Figure S4) and inductively coupled plasma spectroscopy (ICP-OES) (Figure 2f). In agreement with the above described higher stability of particles in milliQ water compared to PBS, particles are more easily separable from PBS than water. While in water $<50 \%$ of the 
MNF-qPDMAEMA(L) and MNF-qPDMAEMA-co-PEGMEMA beads are recovered after 3 minutes of magnetic separation, the recovery rate for the MNF-qPDMAEMA-co-PEGMEMA beads increases to $>>90 \%$ in PBS within the same time. The MNF-qPDMAEMA(L) remain poorly separable even from high ionic strength physiological buffers. Following 10 minutes separation, all MNF particles are recovered from PBS at an efficiency of $>98 \%$. Bare MNF can be completely separated from both milliQ water and PBS within $<3$ minutes using a small neodymium permanent magnet.

In addition to batch magnetic separation experiments, bead capturing was also investigated in a miniaturized flow setting (see ESI, Figure S6). An extracorporeal blood purification device was assembled analogously to a previously constructed magnetic blood purification device ${ }^{5}$ at a scale of $1: 15 .^{29}$ The total extracorporeal circuit

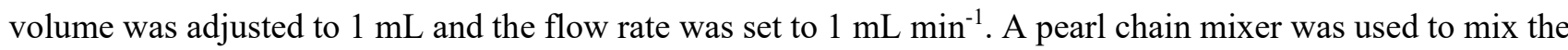
physiological fluid with the MNF suspension. In agreement with the batch extraction experiments, the MNFqPDMAEMA(L) showed higher colloidal stability compared to the MNF-qPDMAEMA-co-PEGMEMA hampering magnetic recovery under flow conditions. In order to achieve quantitative separation of the MNFqPDMAEMA(L), flow rates had to be reduced by a factor of 10 , in order to reach residence times of $>3$ minutes in the magnetic separation unit. Such low flow rates are highly unfavourable in view of clinical translation because of the greatly limited throughput. ${ }^{30}$ The trade-off between high colloidal stability (which is favourable for binding) and efficient magnetic separability is better met by the MNF-qPDMAEMA-co-PEGMEMA, thus making them a potentially superior candidate for continuous magnetic blood purification applications, provided that the capturing efficiency requirements are met.

After characterising colloidal stability as well as separability of the polycationic polymer-functionalized MNF, toxin capturing capacities were investigated as a function of bead concentration within clinically relevant contact times ( $<3$ minutes). In a first experiment, bilirubin capturing was assessed by using an initial bilirubin concentration of $400 \mu \mathrm{M}$, which is equivalent to a plasma concentration of a patient suffering from severe hyperbilirubinemia (Figure 3a). Maximal binding capacities for the respective polymers are displayed in Figure 3b. In physiological buffers, bilirubin capturing efficiencies reached values of $550 \mathrm{mg} / \mathrm{g}$ for MNFqPDMAEMA(L) and $325 \mathrm{mg} / \mathrm{g}$ for MNF-qPDMAEMA-co-PEGMEMA for contact times of 2 minutes. The capturing capacities translate to $35 \%$ and $30 \%$ of the theoretical value for MNF-qPDMAEMA and MNFqPDMAEMA-co-PEGMEMA, respectively (assuming 1:1 coordination of bilirubin with the functional groups 
of the polymer, and no capturing contribution from the PEGMEMA moiety). Binding capacities of the PDMAEMA functionalized magnetic beads are double those of mesoporous carbon particles presented in previous work. ${ }^{31}$ Binding capacities reported for polysulfone ester anion exchanging nanoparticles were $\sim 5-10$ times lower in comparable conditions, despite requiring binding times well above 60 minutes (see also ESI, Table S2). ${ }^{32}$

As a second clinically relevant case, we investigated the removal of heparin. The need to anticoagulate the blood (or plasma) bears a high risk for haemorrhagic complications. ${ }^{33}$ However, the rapid, quantitative removal of unwanted heparin from blood or plasma is not straightforward. The currently available methods of heparin removal, e.g. the administration of protamine, produces potentially lethal side effects. ${ }^{34}$ Therefore, we assessed heparin capturing capacities of the MNF-qPDMAEMA(L) and MNF-qPDMAEMA-co-PEGMEMA. Experiments in Tris- $\mathrm{HCl}$ buffer as well as human serum showed rapid binding kinetics at physiological $\mathrm{pH}$ and high binding capacities for both MNF-qPDMAEMA(L) $(\sim 107 \mathrm{mg} / \mathrm{g})$ and MNF-qPDMAEMA-co-PEGMEMA $(\sim$ $63 \mathrm{mg} / \mathrm{g}$ ) (see also ESI, Table S3). The fact that the beads can be quantitatively recovered from the body fluids enables complete heparin depletion from physiological fluids within minutes as indicated by spectroscopic measurements (Figure 3c,d). Clinically relevant concentrations of heparin can also be efficiently removed from human serum (see ESI, Figure S7). In contrast to previous work, where $\sim 50 \%$ of the heparin could be neutralized in protein rich fluids (human plasma) containing $0.75 \mathrm{IU} / \mathrm{mL}$ of heparin using free polymer chains, ${ }^{35}$ treatment with MNF-qPDMAEMA(L) and MNF-qPDMAEMA-co-PEGMEMA decreased the heparin concentration to baseline levels using MNF concentrations of $100 \mu \mathrm{g} / \mathrm{mL}$ or higher (see ESI, Figure S7).

In addition to efficient toxin capturing, high hemo- and cytocompatibility are essential for future clinical application. Given the documented ability of cationic polymers to agglutinate and/or lyse red blood cells, ${ }^{36,37}$ we measured agglutination and hemolysis at clinically relevant concentrations $(\leq 500 \mu \mathrm{g}$ per $\mathrm{mL})$. While the qPDMAEMA(L)-coated MNF led to severe agglutination of the red blood cells even at concentrations as low as $100 \mu \mathrm{g}$ per $\mathrm{mL}$, no agglutination was observed for MNF-qPDMAEMA-co-PEGMEMA at concentrations of $250 \mu \mathrm{g}$ per $\mathrm{mL}$ and lower (Figure 4a). Hemolysis was below 15\% for all particles investigated (see ESI, Figure S8). However, the severe agglutination observed for MNF-qPDMAEMA(L) could also be a confounding factor leading to a lower apparent hemolytic activity for the qPDMAEMA-coated nanoflowers. In addition to hemol- 
ysis, the cytocompatibility of the polycationic polymer-functionalized MNF was investigated in human monocytes (THP-1 cell line) following exposure for 24 hours (Figure $\mathbf{4 b}$ ). While cytolytic activity was generally high, co-polymerization of DMAEMA with PEGMEMA also reduced cytolytic activity significantly. The addition of PEGMEMA as a co-monomer to DMAEMA yields particles that exhibit significantly lower toxicity towards monocytes, with a lethal concentration (LC50, $24 \mathrm{hrs}$ exposure) that increases from $57 \mu \mathrm{g} / \mathrm{mL}$ (MNFqPDMAEMA) to $121 \mu \mathrm{g} / \mathrm{mL}$ for the MNF-qPDMAEMA-co-PEGMEMA. On the other hand, the introduction of hydrophobic MMA results in toxicity comparable to the homopolymer functionalized MNF (LC50: 62 $\mu \mathrm{g} / \mathrm{mL}$ ) (Figure 4b). In good agreement with previous observations, low polycationic polymer loading not only simplifies the separation process, ${ }^{21}$ but also minimizes cytotoxicity ${ }^{38,39}$ (see ESI, Figure S9), however, critically diminishes capturing efficiencies (see Figure 3a). The much lower cell lysis observed in red blood cells compared to monocytes can be attributed to decreased contact times ( 3 vs. 24 hours) and the inability of red blood cells to endocytose particles. ${ }^{40}$ However, despite the fact that cytolysis could be reduced by introducing PEGMEMA units, the high cytolytic activity suggests extracorporeal application of these nanoparticles in dialysis-type devices, preferably in blood cell depleted plasma, or in the albumin circuit of Molecular Adsorbents Recirculating Systems (MARS) $)^{41,42}$ and Fractionated Plasma Separation, Adsorption and Dialysis systems (FPSA). ${ }^{43,44}$

Polymer-functionalized MNF constitute attractive magnetic blood purification capturing agents as they are accessible by straightforward surfactant free synthesis, exhibit favourable magnetic properties and can readily be surface functionalized with target binding moieties. However, in addition to the high binding capacity and the complete removal of the MNF prior to recirculation of the purified fluid, degradation of potentially incompletely separated MNF is of high interest in order to assess the risks of accidental exposure. Therefore, the differently functionalized nanoparticles were immersed in PBS, acidic citrate buffer (pH 3.0) or lysosomal buffer ( $\mathrm{pH} \sim 4.5$ ) and degradation was measured as a function of time. While in PBS no significant MNF degradation was observed during 14 days $(<5 \mathrm{wt} \%)$, MNF in lysosomal buffer gradually degraded (Figure $4 \mathbf{c}$ ). The polymer brush coatings slightly delayed degradation. Whereas almost $30 \mathrm{wt} \%$ of bare MNF were degraded after one day in lysosomal buffer, only $10 \mathrm{wt} \%$ of the MNF-qPDMAEMA(L) and MNF-qPDMAEMA-coPEGMEMA were dissolved. As expected, degradation in more acidic buffer ( $\mathrm{pH}$ 3.0) led to even faster degradation. The degradation experiments showed that MNF particles are highly stable in PBS for extended periods 
of time, however, gradually degrade in acidic conditions typically encountered in the lysosomal compartment of phagocytic cells. Thus, in the unlikely case of polymer brush functionalized MNF entering the intracorporeal blood circuit, the nanoparticles would be degraded by the body.

The design of optimal capturing materials balances capturing capacity (increased as a function of polymer loading) and magnetic recovery (adversely influenced by high polymer loading). The introduction of PEG moieties attenuates cytolytic effects of the polycationic polymer brushes and improves magnetic recovery, however, also decreases the capturing capacity. The presented MNF-qPDMAEMA-co-PEGMEMA unify sufficient capturing capacity for both bilirubin and heparin at the respective clinically relevant concentrations with favourable magnetic recovery. For the treatment of a patient with $5 \mathrm{~L}$ of blood, a few grams of functionalized MNF would be needed. While scale up of ATRP-based syntheses is not straightforward, first promising steps into this direction have been undertaken, ${ }^{45}$ paving the way for future translation of the approach into practice.

\section{CONCLUSIONS}

Taken together, we demonstrate fast and efficacious toxin removal by qPDMAEMA-based polymers grown from the surface of magnetic nanoflowers exemplified by bilirubin and heparin capturing. Inclusion of PEGMEMA units into the qPDMAEMA polymer brush functionalized MNFs unifies colloidal stability, favourable separability, improved hemocompatibility as well as high binding capacity. While broadband resin-based adsorber cartridges have been successfully applied in preclinical and clinical settings, the unwanted removal of hormones from blood or blood plasma remains a potential shortcoming and requires further investigations focusing on the risk/benefit ratio also for the current magnetic nanoparticle-based system. ${ }^{15}$ Nonetheless, this study presents a highly efficient detoxification system, which may be used for direct body fluid purification or in combination with existing MARS or FPSA systems with the prospect of boosting their capacities. 


\section{SUPPORTING INFROMATION}

Figure S1: High-resolution TEM image of MNF. Figure S2: PDMAEMA and qPDMAEMA polymer mass increase as a function of time. Figure S3: TGA. Figure S4: Sedimentation properties. Figure S5: DLVO calculations. Figure S6: Microfluidic pearl chain mixer. Figure S7: Heparin removal from human serum. Figure S8: Hemolysis data. Figures S9: Cytotoxicity for MNF functionalized with different qPDMAEMA chain lengths. Figures S10: MNF purification. Table S1: CHN data. Table S2: Bilirubin capturing efficiencies. Table S3: Heparin capturing efficiencies.

\section{CONFLICT OF INTEREST}

There are no conflicts of interests to declare.

\section{FUNDING}

This study was funded by Empa.

\section{ACKNOWLEDGEMENTS}

We thank Prof. Hans J. Hug for access to the VSM and Prof. Wendelin J. Stark for access to CHN elemental analysis. 


\section{Materials \& Methods}

Materials. Unfractionated heparin sodium salt $\geq 180 \mathrm{IU} / \mathrm{mg}\left(2 \times 10^{4} \mathrm{~g} / \mathrm{mol}\right)$ was purchased from ROTH, all other materials were purchased from Sigma-Aldrich and used without further purification, where not stated otherwise. The monomers 2-(Dimethylamino)ethyl methacrylate (DMAEMA), methyl methacrylate (MMA), Poly(ethylene glycol) methyl ether methacrylate (PEGMEMA) Mn 300, were passed through a plug of basic alumina (Brockmann Grade I).

Synthesis of Magnetic Nanoflowers. Magnetic nanoflowers (MNF) were synthesized following a surfactant free solvothermal process. ${ }^{23}$ In brief, a $90 \mathrm{~mL}$ teflon lined autoclave was loaded with $60 \mathrm{~mL}$ of ethylene glycol, to which $2.4 \mathrm{~g}$ of $\mathrm{FeCl}_{3} \cdot 6 \mathrm{H}_{2} \mathrm{O}$ and $2.0 \mathrm{~g}$ of $\mathrm{NaHCO}_{3}$ were added. The contents were stirred for 30 min during which bubbling occurred. Then, the autoclave was sealed and heated to $200{ }^{\circ} \mathrm{C}$ for $12 \mathrm{~h}$. The resulting black slurry was magnetically recovered and washed three times with milliQ water $\left(18.2 \mathrm{M} \Omega \mathrm{cm}^{-1}\right)$ followed by three times washing with pure acetone. The clean powder was then vacuum dried at $40^{\circ} \mathrm{C}$ overnight and kept in a sealed flask in the fridge. (Saturation magnetization $\left(\mathrm{M}_{\mathrm{S}}\right) 62 \mathrm{emu} / \mathrm{g}$, mass obtained $0.6 \mathrm{~g}$ ).

Functionalization of MNF with polydopamine (PDA). To $110 \mathrm{~mL}$ of a $10 \mathrm{mM}$ Tris- $\mathrm{HCl}$ buffer of $\mathrm{pH} 8.45$, $0.2 \mathrm{~g}$ of dry MNF were added. The resulting mixture was sonicated until the MNF were homogenously dispersed. Then, $5 \mathrm{~mL}$ of a freshly prepared solution of $40 \mathrm{mg} / \mathrm{mL}$ of dopamine- $\mathrm{HCl}$ in Tris- $\mathrm{HCl}$ buffer were added to the particle dispersion under sonication. The resulting mixture was sonicated for $5 \mathrm{~min}$ and then transferred to an orbital shaker, where it was left to react for exactly 1 hour since addition of dopamine. The products of the reaction were magnetically separated and the supernatant decanted. The particles were washed three times with milliQ water and three times with pure methanol, followed by vacuum drying at $40^{\circ} \mathrm{C}$ overnight. (FTIR $\left.1486 \mathrm{~cm}^{-1}, 1266 \mathrm{~cm}^{-1}\right)$.

Functionalization of PDA modified MNF with 2-Bromoisobutyryl Bromide. A round bottom flask was loaded with $120 \mathrm{~mL}$ of dry toluene. To that, $0.2 \mathrm{~g}$ of polydopamine modified MNF were added and the resulting mixture was sonicated until particles were well dispersed. The mixture was then cooled to $0^{\circ} \mathrm{C}$ while still sonicating using an ice bath. Then, $475 \mu \mathrm{L}$ of triethylamine followed by $390 \mu \mathrm{L}$ of 2-bromoisobutyryl bromide were added. The reaction mixture was then sealed and left to react overnight at room temperature on an orbital shaker. The modified particles were then magnetically recuperated and washed three times with toluene, three times 
with dichloromethane and finally twice in acetone. The resulting slurry was vacuum dried at $40^{\circ} \mathrm{C}$ overnight. (TGA: 5.9\% mass increase, Elemental analysis: C 5.15\% H 0.68\% N 0.57\% Br 1.25\%).

SI-ATRP of DMAEMA from MNF-PDA-Br. The protocol for the SI-ATRP was adjusted from Dong et al. ${ }^{24}$ In brief, $50 \mathrm{mg}$ of MNF-PDA-Br particles were loaded into a glass vial, followed by the addition of $2 \mathrm{~mL}$ of pure acetone, $27 \mu \mathrm{L}$ of HMTETA ligand and $5.6 \mathrm{~mL}$ of DMAEMA purified monomer. The contents of the vial were sonicated until particles were fully dispersed. The vial was then transferred to a Schlenk tube which was immediately sealed and submitted to three freeze pump thaw cycles. To a second Schlenk tube $2.2 \mathrm{mg}$ of $\mathrm{CuCl}_{2}$ and $8.8 \mathrm{mg}$ of $\mathrm{CuCl}$ were added, the resulting system was sealed and then evacuated and back-filled with nitrogen four times. The reaction was started by cannulating the contents of the first Schlenk tube to the second, the contents were briefly sonicated to dissolve the copper salts and the system was then left to react at $40^{\circ} \mathrm{C}$ under vigorous shaking. The reaction was stopped by transferring the contents of the vessel to pure acetone. The resulting particles were magnetically separated and the supernatant discarded. The particle slurry was then washed six times with acetone and dried in vacuo overnight. For the copolymerization of DMAEMA with PEGMEMA or MMA the volume of DMAEMA was decreased by a third and replaced with the corresponding monomer. (FTIR: $1716 \mathrm{~cm}^{-1}(\mathrm{C}=\mathrm{O}), 2763 \mathrm{~cm}^{-1}$ and $2939 \mathrm{~cm}^{-1}(\mathrm{C}-\mathrm{H}), 1142 \mathrm{~cm}^{-1}(\mathrm{C}-\mathrm{N})$ ).

Quaternization of DMAEMA brushes. The quaternization of the PDMAEMA polymer brush functionalized MNF was done using methyl iodide (MeI) by adjusting a protocol described previously. ${ }^{24}$ More specifically, a glass vial was loaded with $20 \mathrm{mg}$ of MNF-PDMAEMA particles. Then, $2.25 \mathrm{~mL}$ of pure 2-propanol and 2.25 $\mathrm{mL}$ of acetone were added and the contents were sonicated. Using a syringe, $500 \mu \mathrm{L}$ of MeI were then added and the reaction mixture was kept under sonication for 15 minutes before it was sealed and moved to an orbital shaker for 24 hours. The particles were then magnetically recuperated and washed three times with pure acetone and three times with pure isopropanol, before dried in-vacuo overnight. Prior to use, particles were washed with PBS and magnetically recovered to remove ultra-small clusters and copper residues coming from the SI-ATRP (see ESI Figure S10).

Transmission electron microscopy (TEM). The MNF particles were investigated using a JEOL 2200FS TEM operated at $200 \mathrm{kV}$ for imaging, high resolution imaging (HR-TEM) and selected area electron diffraction (SAED). milliQ water dispersions containing $1 \mathrm{mg} / \mathrm{mL}$ MNF particles were drop-casted onto a carbon coated copper grid (200 mesh) and left to evaporate. Particle diameters $\left(\mathrm{d}_{\mathrm{TEM}}\right)$ were determined manually based on 
TEM image analysis in Image (Version ImageJ 1.52a, National Institutes of Health, USA).

X-Ray powder diffraction (XRD). The analysis of the crystal structure was performed in the Debye Scherrer configuration using a Panalytical $\mathrm{X}^{\prime} \mathrm{Pert}^{3}$ Powder diffractometer equipped with a sealed $\mathrm{Cu}$-Tube (wavelengths $\mathrm{K} \alpha 1, \lambda=1.54056 \AA$ and $\mathrm{K} \alpha 2, \lambda=1.54439 \AA)$ and a Ni filter removing the contribution of the characteristic $\mathrm{K} \beta(\lambda$ $=1.3922 \AA$ ) rays. To converge the beam onto the rotating quartz capillary containing the sample at the goniometer centre a $1^{\circ}$ divergence slit, a focusing X-ray mirror, a $20 \mathrm{~mm}$ mask and a $1 / 2^{\circ}$ anti-scattering slit were used. A PIXcel1D detector collected the diffraction patterns from $20^{\circ}$ to $100^{\circ} 2 \theta$. For crystallite size determination, Diffrac.Eva software (DIFFRAC.EVA, Version 4.2.1.10) was used to apply the Scherrer equation to the 5 most intense peaks (angles $\left.2 \theta(\mathrm{hkl}): 30.049^{\circ}(220) ; 35.379^{\circ}(311) ; 42.971^{\circ}(400) ; 56.959^{\circ}(333 / 511) ; 62.497^{\circ}(440)\right)$ and taking the average, after automatic background subtraction and k 22 stripping.

Vibrating Sample Magnetometry. Vacuum-dried MNF $(20 \mathrm{mg})$ were put into polymer-sample holders and analyzed by vibrating sample magnetometry (VSM) using the physical properties measurement system PPMS (PPMS, Version P525, Quantum Design GmbH, Germany ) of Quantum Design with a maximum magnetic field of $5 \mathrm{~T}$.

CHN elemental analysis. Samples dried in vacuum for 48 hours were ground and carbon, hydrogen, nitrogen and bromide were analyzed using a LECO Truspec Micro instrument (Leco Corporation, Michigan, USA). The micro analyzer was calibrated using sulfanilamide. All samples were measured in duplicates using a standard program of $2 \mathrm{mg}$ of powder that is subsequently oxidized/pyrolized over 70 seconds.

FTIR. Infrared absorption spectra were measured using Varian 640-IR spectrometer equipped with diamond attenuated total reflectance (ATR) optics from previously dried powders.

Thermogravimetric analysis (TGA). All samples were dried in vacuum prior to measurement and loaded into ceramic crucibles with masses ranging from 2-5 mg. Using using a NETZSCH TG 209 F1 instrument (NETZSCH-Gerätebau $\mathrm{GmbH}$, Selb, Germany) ramping was set at $10^{\circ} \mathrm{C}$ per minute and all analysis met the final temperature of $900^{\circ} \mathrm{C}$ under nitrogen.

DLS and Zeta potential. Hydrodynamic size measurements as well as zeta potential measurements were performed using a dynamic light scattering (DLS) ZetaSizer90 instrument from Malvern at $0.1 \mathrm{mg} / \mathrm{mL}$ particle concentrations, using the refractive index of iron oxide $(n=2.918)$. Particle suspensions were prepared by dilution from a $1 \mathrm{mg} / \mathrm{mL}$ stock and were briefly sonicated for 30 seconds before measurement. Stabilization time 
was set to 30 seconds. Zeta potential measurements were performed using a background of $1 \%$ PBS and stabilization time was equally set at 30 seconds.

Particle sedimentation. Sedimentation experiments of the as prepared MNF were performed using a Jenway $6705 \mathrm{UV} / \mathrm{Vis}$ spectrometer, in analogy to experiments performed by Keevend et al. ${ }^{46}$ More specifically, sedimentation kinetics were measured using $1.5 \mathrm{~mL}$ PMMA cuvettes and by monitoring the top $3 \mathrm{~mm}$ of the particle suspension. Prior to measurements the linear concentration range was determined at $405 \mathrm{~nm}$ (concentrations: 0 , $31.3,62.5,125, \mu \mathrm{g} / \mathrm{mL})$. The total volume used in the measurements was $610 \mu \mathrm{L}$. The kinetic absorbance measurements were performed for 2 hours continuously using an initial MNF concentration of $100 \mu \mathrm{g} / \mathrm{mL}$. Afterwards, absorbance at $405 \mathrm{~nm}$ was measured at time points 4,18 , and 24 hours for dispersions that had not completely sedimented. DLVO calculations were performed based on methods described previously. ${ }^{16}$

Magnetic Separability. In order to define the timescales of separation of bare as well as surface functionalized MNF particles, suspensions of particles previously washed three times in milliQ water and three times in PBS were prepared at a concentration of $0.25 \mathrm{mg} / \mathrm{mL}$, in $1.5 \mathrm{~mL}$ Eppendorf tubes. The tubes were then placed tip down and for a defined amount of time on a $4.5 \times 3 \times 1 \mathrm{~cm}^{3}$ neodymium magnet, using a Styrofoam holder to keep them in contact with the magnet. An aliquot of $120 \mu \mathrm{L}$ was collected from the supernatant and measured using a plate reader (Mithras2 LB 943, Berthold Technologies, Bad Wildbad, Germany) at $405 \mathrm{~nm}$ as well as analyzed by ICP-OES (Agilent 5110, Santa Clara, CA, USA). For continuous magnetic particle separation experiments, a microfluidic pearl chain mixer was used (microfluidic chipshop, Jena, Germany). ${ }^{5}$ MNF suspensions of 1 $\mathrm{mg} / \mathrm{mL}$ were fed into one inlet and the physiological fluid was fed into the second inlet at equal flow rates (1 $\mathrm{mL} / \mathrm{min}$ ). The total fluid volume between injection point and magnetic separation unit (two permanent magnets) was adjusted to $1 \mathrm{~mL}$. Particle separation was monitored optically by UV/Vis.

Bilirubin removal. For bilirubin capturing experiments, $50 \mathrm{mg}$ of bilirubin were dissolved in $0.1 \mathrm{M} \mathrm{NaOH}$ solution to yield a dark orange solution $(17.1 \mathrm{mM})$. Then, $470 \mu \mathrm{L}$ of this solution were added to $9.0 \mathrm{~mL}$ of PBS and the $\mathrm{pH}$ was adjusted with a $1 \mathrm{M} \mathrm{NaOH}$ solution to $\mathrm{pH} 7.8$ and a final volume of $10 \mathrm{~mL}$. The solution was then diluted to yield an $800 \mu \mathrm{M}$ stable bilirubin solution. Particles previously washed three times in PBS were dispersed in PBS. The suspensions were then mixed in equal parts with the bilirubin solution to yield a final bilirubin concentration of $400 \mu \mathrm{M}$. After vortexing the particle solutions for $10 \mathrm{sec}$, tubes were kept for $2 \mathrm{~min}$ before particles were separated using a small permanent magnet. $120 \mu \mathrm{L}$ of the supernatant were transferred to 
a 96-well plate and the absorbance was measured at $405 \mathrm{~nm}$ using a plate reader (Mithras2 LB 943, Berthold Technologies, Bad Wildbad, Germany).

Heparin removal. The heparin removal experiment was adapted from Välimäki et al. ${ }^{35}$ More specifically, a freshly prepared $50 \mathrm{mM}$ Tris- $\mathrm{HCl}$ buffer solution at $\mathrm{pH} 7.3$ was used to prepare a stock solution of $0.5 \mathrm{mg} / \mathrm{mL}$ of heparin and a methylene blue (MB) stock solution of $0.064 \mathrm{mg} / \mathrm{mL}$. These solutions were then mixed in a 1:4 volume ratio respectively and then further diluted to yield a stock solution of $0.086 \mathrm{mg} / \mathrm{mL}$ of $\mathrm{MB}$ and 0.16 $\mathrm{mg} / \mathrm{mL}$ of heparin. MNF suspensions were prepared by suspending previously washed MNF in Tris-HCl buffer at a concentration of $2 \mathrm{mg} / \mathrm{mL}$. Subsequently, $200 \mu \mathrm{L}$ of the Heparin-MB solution was added to a $1.5 \mathrm{~mL}$ Eppendorf tube. An appropriate amount of particles and Tris-HCl buffer were added in order to reach the final volume of $400 \mu \mathrm{L}$, and a final concentration of $0.08 \mathrm{mg} / \mathrm{mL}$ of heparin and $0.043 \mathrm{mg} / \mathrm{mL}$ of MB. Particle concentrations were chosen as $1,0.5,0.25,0.1,0.05$, and $0.025 \mathrm{mg} / \mathrm{mL}$ and particle-heparin/MB solutions were briefly vortexed and left to stand for $2 \mathrm{~min}$, before MNF were magnetically separated. The residual supernatant was sampled in triplicates $(120 \mu \mathrm{L})$ and distributed on a 96-well plate. Absorbance was measured at $664 \mathrm{~nm}$ and $568 \mathrm{~nm}$ using a plate reader (Mithras2 LB 943, Berthold Technologies, Bad Wildbad, Germany).

Agglutination and Hemolysis. For agglutination and hemolysis experiments, whole blood from healthy volunteers was collected in tubes containing 0.109 M sodium citrate after obtaining written informed consent (ethical approval, EKSG 12/111). Tubes were centrifuged at $1000 \times \mathrm{g}$. The plasma was discarded and red blood cells were washed in PBS three times. The hemoglobin concentration was adjusted to $\sim 30 \mathrm{mg} / \mathrm{mL}$. Blood cell suspensions were then incubated with the differently functionalized nanoparticles. Triton X (1\%) served as positive and PBS as negative control. After 15 minutes of incubation, $10 \mu \mathrm{L}$ of each sample were transferred to a glass slide and agglutination was imaged using an optical microscope. After incubation for $3 \mathrm{~h}$ at $37^{\circ} \mathrm{C}$, the samples were centrifuged at $6000 \times \mathrm{g}$ for $10 \mathrm{~min}$. Supernatants were transferred to a 96 -well plate and absorption at 570 nm (hemoglobin) was measured using a plate reader (Mithras2 LB 943, Berthold Technologies, Bad Wildbad, Germany). Interference due to interactions of free haemoglobin and the nanoparticles has been excluded by measuring haemoglobin sorption on the particles.

LDH Assay. In order to investigate the cytotoxicity of the produced nanoparticles, human monocytes (THP-1), derived from an acute monocytic leukemia patient, were cultured in RPMI- 1640 medium at $37{ }^{\circ} \mathrm{C}$ with $10 \%$ 
FBS and under a 5\% $\mathrm{CO}_{2}$ humidified atmosphere. Using a 48-well plate 100000 cells were seeded in $225 \mu \mathrm{L}$ of $10 \%$ FBS containing medium. In order to bring particles in contact with cells, previously three times washed in PBS particle suspensions were prepared in PBS and $25 \mu \mathrm{L}$ aliquots were added to cells to give concentrations ranging from 0 to $1000 \mu \mathrm{g} / \mathrm{mL}$. Total volume in each well was $250 \mu \mathrm{L}$, PBS was used as the negative control and Triton-X100 (1\%) as the positive one. The nanoparticles were left to incubate on the cells for 24 hours at $37{ }^{\circ} \mathrm{C}$ with $10 \% \mathrm{FBS}$ and under a $5 \% \mathrm{CO}_{2}$ humidified atmosphere. After incubation the particles and cells were removed from the supernatant by centrifugation at $2250 \times \mathrm{g}$ for $15 \mathrm{~min}$ at ambient temperature. $50 \mu \mathrm{L}$ per well of every well were transferred to a flat bottom 96 well plate, while keeping a magnet under the plate of origin to avoid resuspension. To each well were then added $50 \mu \mathrm{L}$ of substrate mix from Promega CytoTox 96 NonRadioactive cytotoxicity assay kit. Plates were then incubated in the dark at room temperature for 15 min and the absorbance was measured at $490 \mathrm{~nm}$ using a plate reader (Mithras2 LB 943, Bad Wildbad, Germany). The data were analyzed by subtracting the negative control average values (medium, PBS and LDH reagent) from all absorbance values. Values are expressed relative to the positive control. LC50 values were calculated using Origin Pro (Version 2018 SR1, OriginLab Corporation, Northhampton, MA, USA) and a dose response sigmoidal curve fit.

Degradability. The degradation of the nanoparticles was adapted from previously published literature . More $^{5}$ precisely the dissolved iron content was measured at both $\mathrm{pH} 4.5$ (mimicking lysosomal conditions) and at $\mathrm{pH}$ 3 in $10 \mathrm{mM}$ citrate buffer solution, as well as at $\mathrm{pH} 7.3$ (phosphate buffered saline). Suspensions of $50 \mu \mathrm{g} / \mathrm{mL}$ were loaded into $2 \mathrm{~mL}$ Eppendorf tubes $(0.5 \mathrm{~mL}$ final volume) and incubated horizontally on an orbital shaker for a predetermined amount of time (days: 1, 2, 3, 7, 10, 14). Following particle separation by high speed centrifugation $\left(20^{\prime} 000 \times \mathrm{g}\right.$ for 20 mins) and magnetic separation $250 \mu \mathrm{L}$ were aliquoted and further acidified with $250 \mu \mathrm{L}$ of ultrapure $37 \% \mathrm{HCl}$ and then diluted to a final volume of $5 \mathrm{~mL}$, degradation of the MNF particles was determined by measuring the iron content in the supernatant using ICP-OES (Agilent 5110, Santa Clara, CA, USA).

Copper Content. The content of copper in the polymer brush was evaluated using ICP-MS (Agilent 7900, Santa Clara, CA, USA). More precisely suspensions of $1 \mathrm{mg} / \mathrm{mL}$ of MNF-qPDMAEMA particles were made using the dried product powder after quaternization. The particles were dispersed in milliQ water or PBS and aliquoted $(25 \mu \mathrm{L})$ after $0,1,3,5$ washes with milliQ water or PBS. The particle dispersions were then added 
$225 \mu \mathrm{L}$ of ultrapure $37 \% \mathrm{HCl}$ and then diluted to a final volume of $5 \mathrm{~mL}$. Particle suspensions at the level of the 0 wash were also washed with a 1:1:1 mixture of milliQ:acetone:acetylacetone (see ESI, Figure S10) adjusted from a previous study. ${ }^{47}$ 


\section{FIGURES}

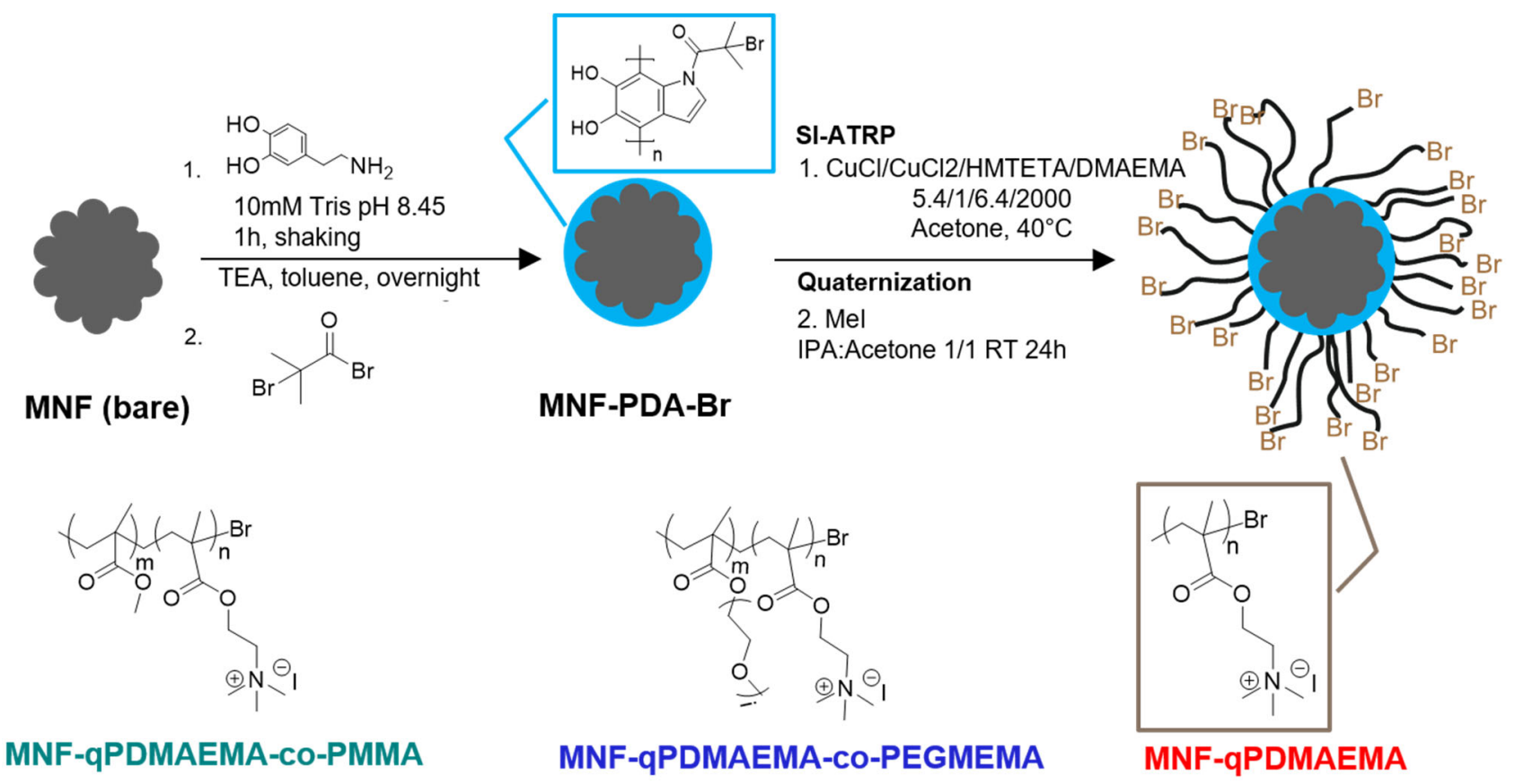

Scheme 1: Reaction scheme. Magnetic nanoflowers (MNF) were functionalized with a polydopamine (PDA)based atom transfer radical polymerization (ATRP) initiator. Then, MNF were functionalized with poly(2-dimethylaminoethyl methacrylate) (PDMAEMA)-based polymer brushes. Amines were quaternized. In addition to qPDMAEMA homo-polymers, co-polymers with poly(ethylene glycol) methyl ether methacrylate (PEGMEMA) or poly(methyl methacrylate) (PMMA) were synthesized analogously. 

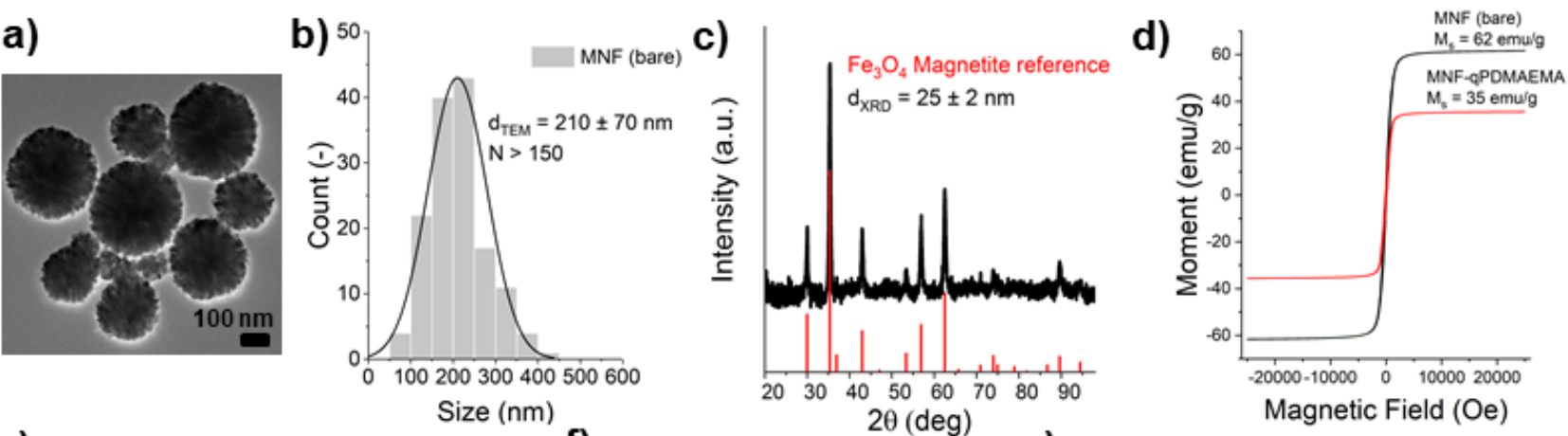

e)

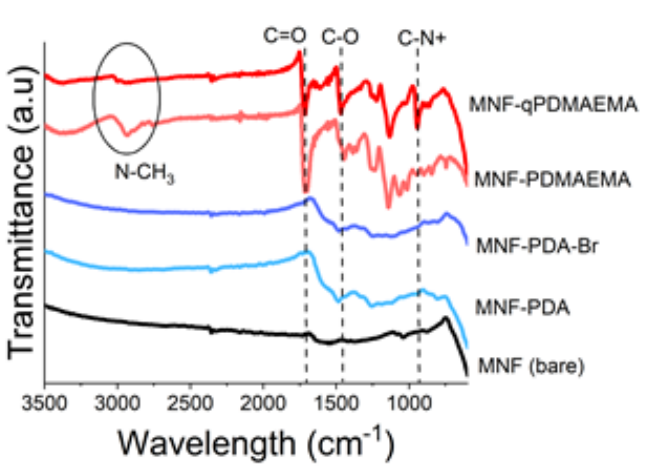

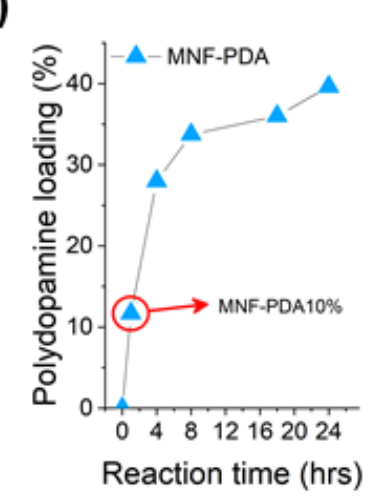

g)

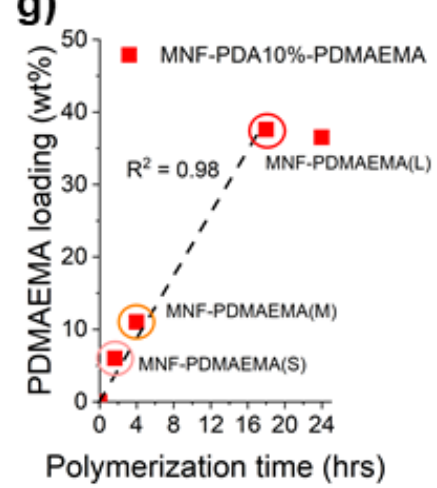

Figure 1: (a) Bright field transmission electron micrograph of as-prepared MNF (bare) with (b) corresponding

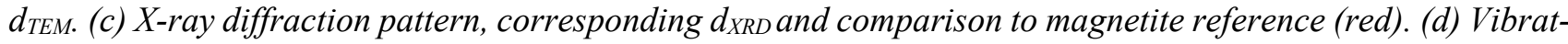
ing sample magnetometry (VSM) hysteresis curves and corresponding saturation magnetization values $\left(M_{s}\right)$ for bare and functionalized MNF. (e) Fourier transform infrared spectra for MNF (bare), MNF-PDA, MNF-PDABr, MNF-PDMAEMA and MNF-qPDMAEMA. (f) PDA loading on the MNF as a function of reaction time, red circle: sample used for further functionalization with PDMAEMA. (g) PDMAEMA loading as a function of reaction time (including a linear fit). Circles indicate particles with different PDMAEMA content used in further experiments. 

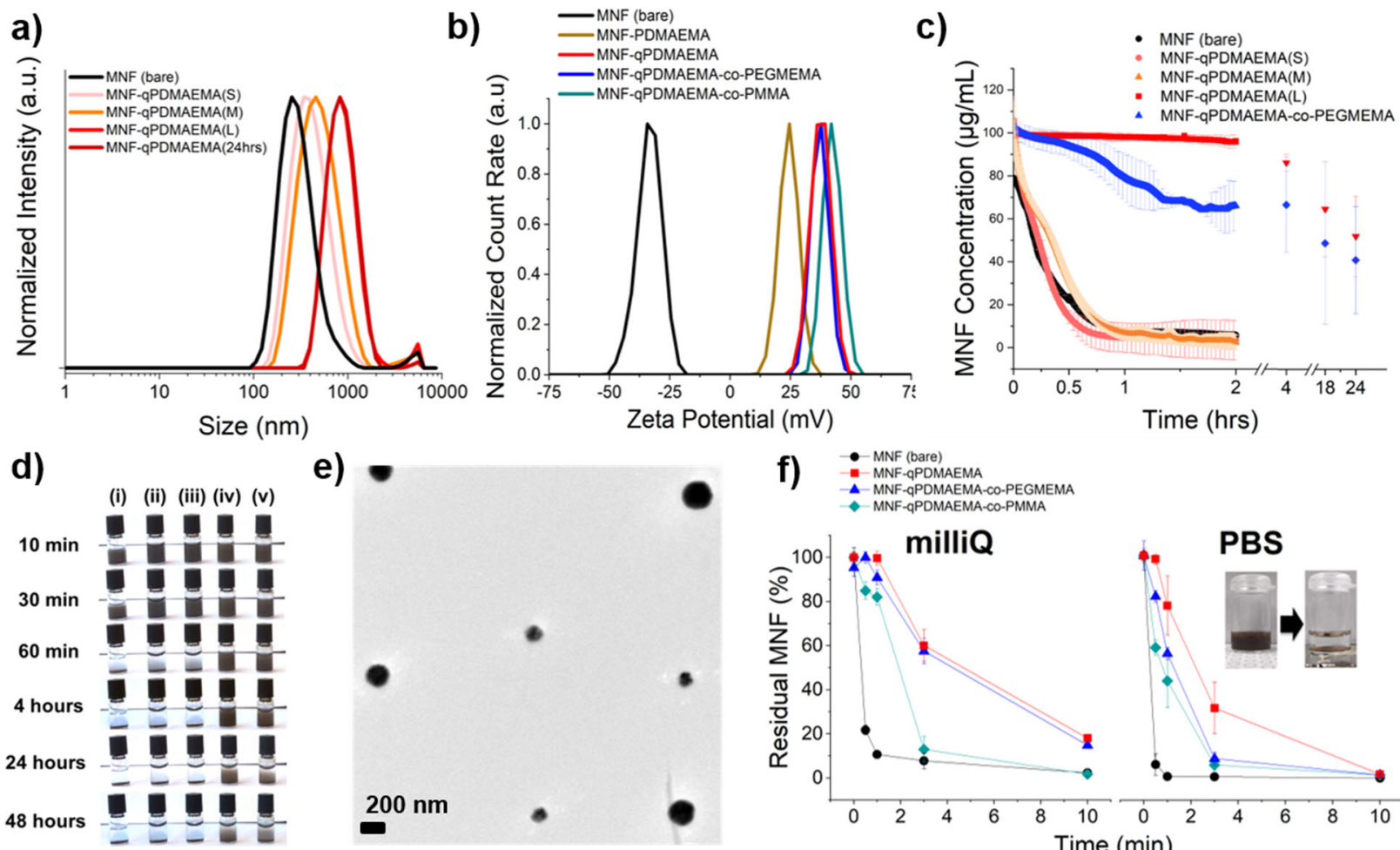

e)

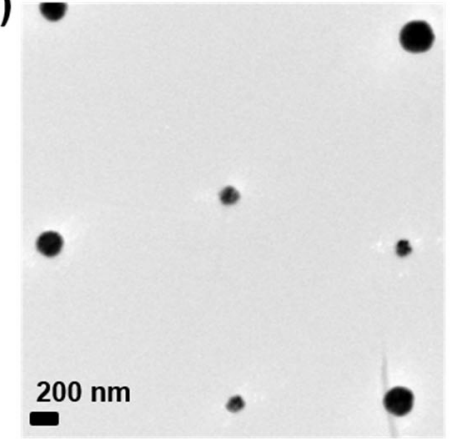

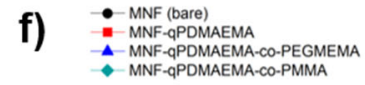

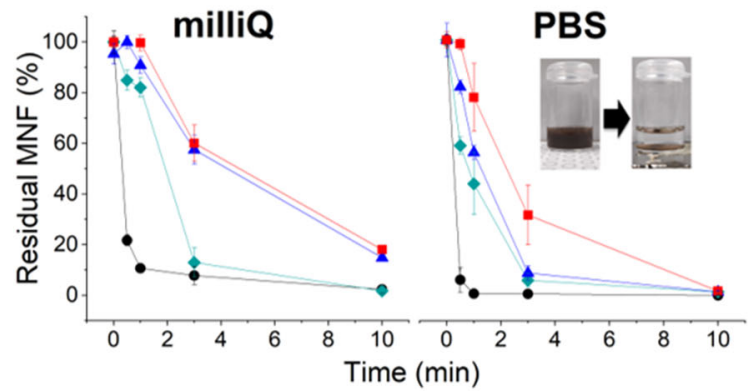

Figure 2: (a) Hydrodynamic diameter as a function of polymerization time for MNF-qPDMAEMA. (b) Zeta potential for bare $M N F, M N F-P D M A E M A, M N F-q P D M A E M A(L)$ and $M N F-q P D M A E M A-c o-P E G M E M A$. (c) Sedimentation of the differently functionalized nanoparticles monitored by UV/Vis spectroscopy for 2 hours. Incompletely sedimented samples were analysed for up to 24 hours. (d) Corresponding photographs of nanoparticle dispersions (initial MNF concentration: $0.25 \mathrm{mg} / \mathrm{mL}$, in PBS) over a period of 48 hours. (i) MNF (bare), (ii) $M N F-q P D M A E M A(S)$, (iii) $M N F-q P D M A E M A(M)$, (iv) $M N F-q P D M A E M A(L)$, (v) $M N F-q P D M A E M A-c o-$ PEGMEMA. (e) Transmission electron micrograph of MNF-qPDMAEMA(L) isolated from suspension. (f) Magnetic separability in milliQ water and PBS as a function of magnetic separation time. Residual MNF concentrations determined by ICP-OES. Error bars show standard deviations $(N=3$, three independent experiments). 
a)

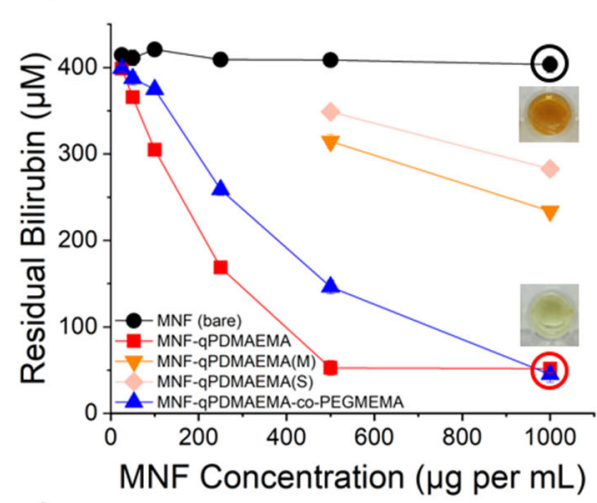

c)

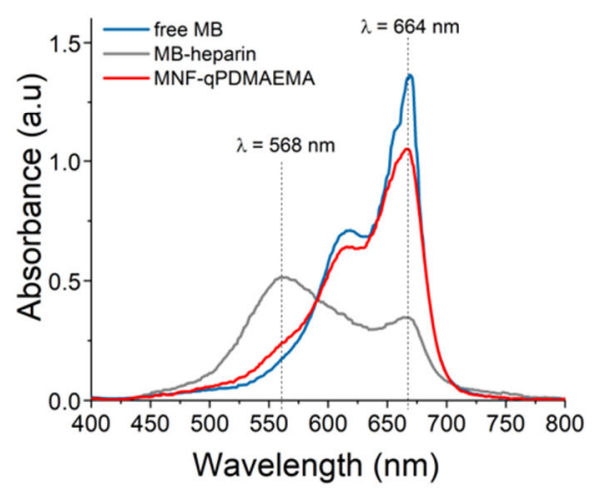

b)

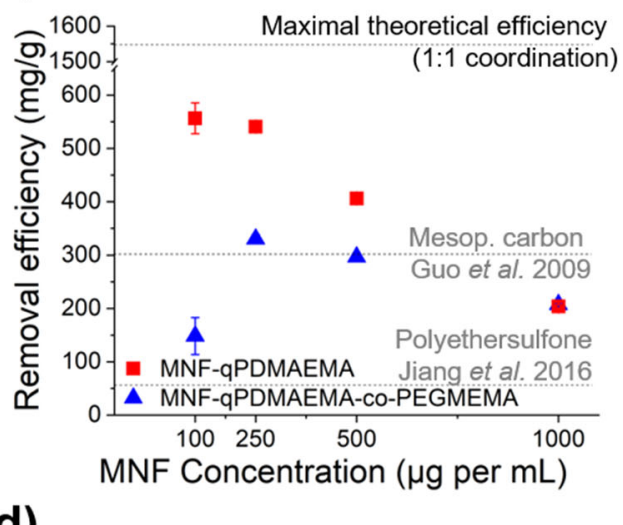

d)

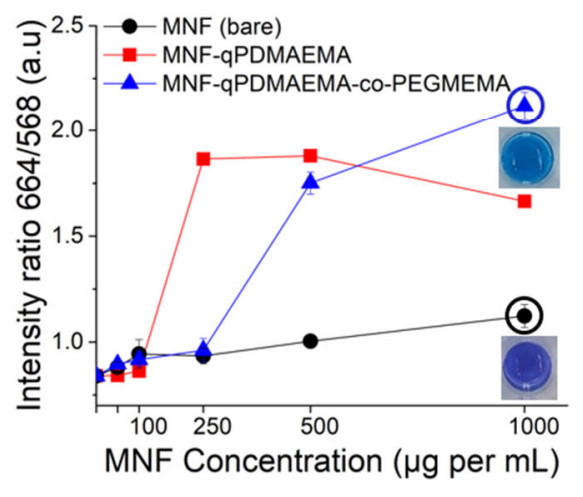

Figure 3: (a) Bilirubin capturing from physiological buffers using a clinically relevant initial concentration of $400 \mu M$. Effects of bead functionalization (including polymer composition and loading) and bead concentration using contact times of 2 minutes. Circles indicating the difference of residual bilirubin at $1 \mathrm{mg} / \mathrm{mL}$ between bare and functionalized MNF. (b) Bilirubin capturing efficiencies as a function of particle concentration. Comparison to efficiencies published in the literature (dotted lines) using alternative sorbents and identical experimental conditions. (c) UV/Vis spectra of free methylene blue (MB), heparin bound MB (MB-heparin) and MNFqPDMAEMA(L) treated MB-heparin. (d) Peak ratios used to monitor change between MB-heparin and free heparin, indicating the capturing of the heparin by MNF-qPDMAEMA-co-PEGMEMA. Circles indicate the difference of colour due to heparin removal between bare and functionalized MNF (1 mg/mL). Error bars show standard deviations ( $N=3$, three independent experiments). 
a)
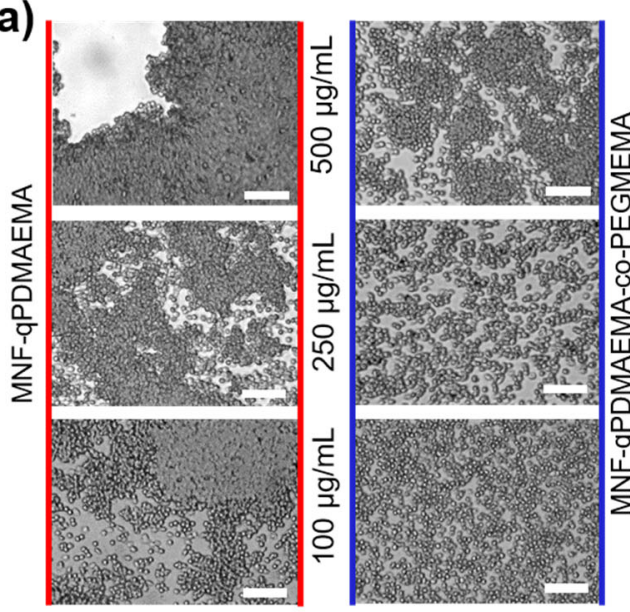

b)

c)

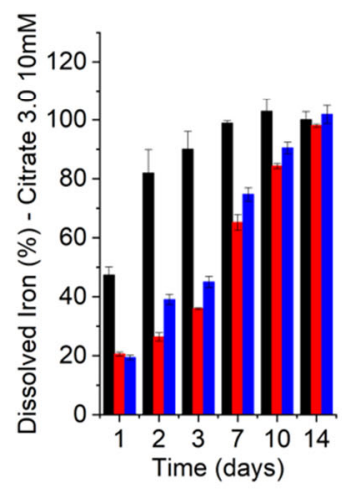

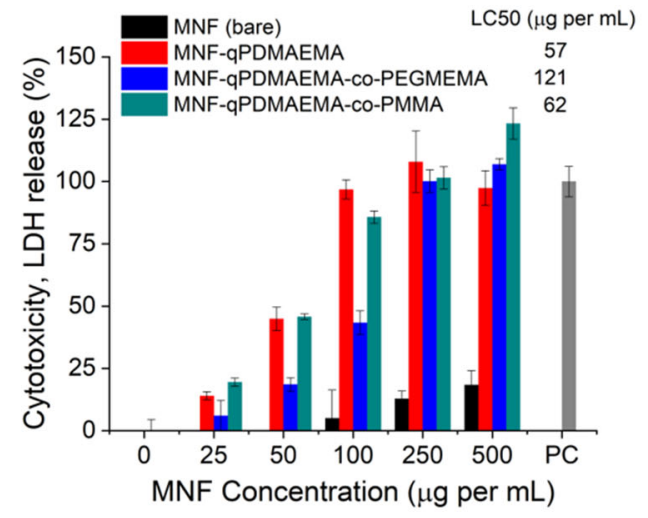

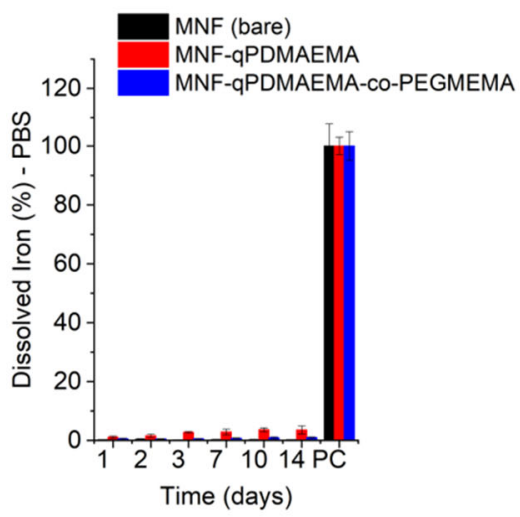

Figure 4: (a) Optical micrographs illustrating red blood cell agglutination for MNF-qPDMAEMA(L) (left). Scale bar: $100 \mu \mathrm{m}$. The agglutination effect is less pronounced for MNF-qPDMAEMA-co-PEGMEMA (right) with no observed agglutination for concentrations $\leq 250 \mu \mathrm{g} / \mathrm{mL}$. (b) Lactate dehydrogenase release from human monocytes (THP-1) exposed to MNF-qPDMAEMA(L) and its co-polymers with PEGMEMA and PMMA for 24 hours. Values are expressed relative to the positive control (PC, Triton X-100, full cell lysis). (c) MNF degradability measured in acidic citrate buffer ( $p H$ 3), artificial lysosomal buffer ( $p H 4.5)$ and PBS over time. PC indicates fully chemical digestion of the MNF samples. Error bars indicate standard deviations $(N=3$, three independent experiments). 


\section{References}

(1) Bernal, W.; Wendon, J. Acute Liver Failure. N. Engl. J. Med. 2013, 369 (26), 2525-2534. https://doi.org/10.1056/NEJMra1208937.

(2) Lee, W. M.; Squires Jr, R. H.; Nyberg, S. L.; Doo, E.; Hoofnagle, J. H. Acute Liver Failure: Summary of a Workshop. Hepatology 2008, 47 (4), 1401-1415. https://doi.org/10.1002/hep.22177.

(3) Bernal, W.; Auzinger, G.; Dhawan, A.; Wendon, J. Acute Liver Failure. The Lancet 2010, 376 (9736), 190-201. https://doi.org/10.1016/S0140-6736(10)60274-7.

(4) Pless, G. Artificial and Bioartificial Liver Support. Organogenesis 2007, 3 (1), 20-24.

(5) Bohmer, N.; Demarmels, N.; Tsolaki, E.; Gerken, L.; Keevend, K.; Bertazzo, S.; Lattuada, M.; Herrmann, I. K. Removal of Cells from Body Fluids by Magnetic Separation in Batch and Continuous Mode: Influence of Bead Size, Concentration, and Contact Time. ACS Appl. Mater. Interfaces 2017, 9 (35), 29571-29579. https://doi.org/10.1021/acsami.7b10140.

(6) Kang, J. H.; Super, M.; Yung, C. W.; Cooper, R. M.; Domansky, K.; Graveline, A. R.; Mammoto, T.; Berthet, J. B.; Tobin, H.; Cartwright, M. J.; Rottman, M.; Waterhouse, A.; Mammoto, A.; Gamini, N.; Rodas, M. J.; Kole, A.; Jiang, A.; Valentin, T. M.; Diaz, A.; Takahashi, K.; Ingber, D. E. An Extracorporeal Blood-Cleansing Device for Sepsis Therapy. Nat. Med. 2014, 20 (10), 1211-1216. https://doi.org/10.1038/nm.3640.

(7) Lattuada, M.; Ren, Q.; Zuber, F.; Galli, M.; Bohmer, N.; T. Matter, M.; Wichser, A.; Bertazzo, S.; B. Pier, G.; K. Herrmann, I. Theranostic Body Fluid Cleansing: Rationally Designed Magnetic Particles Enable Capturing and Detection of Bacterial Pathogens. J. Mater. Chem. B 2016, 4 (44), 7080-7086. https://doi.org/10.1039/C6TB01272H.

(8) Falkenhagen, D.; Strobl, W.; Vogt, G.; Schrefl, A.; Linsberger, I.; Gerner, F. J.; Schoenhofen, M. Fractionated Plasma Separation and Adsorption System: A Novel System for Blood Purification to Remove Albumin Bound Substances. Artif. Organs 1999, 23 (1), 81-86. https://doi.org/10.1046/j.15251594.1999.06292.x.

(9) Kang, J. H.; Um, E.; Diaz, A.; Driscoll, H.; Rodas, M. J.; Domansky, K.; Watters, A. L.; Super, M.; Stone, H. A.; Ingber, D. E. Optimization of Pathogen Capture in Flowing Fluids with Magnetic Nanoparticles. Small 2015, 11 (42), 5657-5666. https://doi.org/10.1002/smll.201501820.

(10) Herrmann, I. K.; Urner, M.; Koehler, F. M.; Hasler, M.; Roth-Z'Graggen, B.; Grass, R. N.; Ziegler, U.; Beck-Schimmer, B.; Stark, W. J. Blood Purification Using Functionalized Core/Shell Nanomagnets. Small 2010, 6 (13), 1388-1392. https://doi.org/10.1002/smll.201000438.

(11) Cai, K.; Li, J.; Luo, Z.; Hu, Y.; Hou, Y.; Ding, X. $\beta$-Cyclodextrin Conjugated Magnetic Nanoparticles for Diazepam Removal from Blood. Chem. Commun. 2011, 47 (27), 7719-7721. https://doi.org/10.1039/C1CC11855B.

(12) Herrmann, I. K.; Schlegel, A. A.; Graf, R.; Stark, W. J.; Beck-Schimmer, B. Magnetic Separation-Based Blood Purification: A Promising New Approach for the Removal of Disease-Causing Compounds? $J$ Nanobiotechnology 2015, 13, 49-49. https://doi.org/10.1186/s12951-015-0110-8.

(13) Maini, R.; Gaylor, J. D. S.; Courtney, J. M.; Wozniak, A. Removal Of Protein-Bound Bilirubin From Plasma Using Small Particle Size Anion Exchange Resin And Microfiltration. In Biomaterials in Artificial Organs: Proceedings of a seminar on biomaterials held at the University of Strathclyde, Glasgow in September 1983; Paul, J. P., Gaylor, J. D. S., Courtney, J. M., Gilchrist, T., Eds.; Palgrave Macmillan UK: London, 1984; pp 186-190. https://doi.org/10.1007/978-1-349-07283-5_24.

(14) Lopukhin, U. M.; Shurkalin, B. K.; Leykin, U. A.; Evseev, N. G.; Molodenkov, M. N.; Kuznetzov, V. N.; Gorchakov, V. D.; Blagosklonov, A. S. Bilirubin Removal by Anion Exchange Resin. The Lancet 1975, 306 (7932), 461. https://doi.org/10.1016/S0140-6736(75)90880-6.

(15) Brunner, G.; Mito, M. Artificial Liver Support: Concepts, Methods, Results; Springer Science \& Business Media, 2012.

(16) Uzun, L.; Denizli, A. Bilirubin Removal Performance of Immobilized Albumin in a Magnetically Stabilized Fluidized Bed. J. Biomater. Sci., Polymer Ed. 2006, 17 (7), 791-806.

https://doi.org/10.1163/156856206777656481. 
(17) Ma, C.-F.; Gao, Q.; Zhou, J.; Chen, Q.-X.; Han, B.; Xia, K.-S.; Zhou, C.-G. Facile One-Pot Synthesis of Magnetic Nitrogen-Doped Porous Carbon for High-Performance Bilirubin Removal from BSA-Rich Solution. RSC Adv. 2017, 7 (4), 2081-2091. https://doi.org/10.1039/C6RA25027K.

(18) Wei, H.; Xu, L.; Ren, J.; Jia, L. Adsorption of Bilirubin to Magnetic Multi-Walled Carbon Nanotubes as a Potential Application in Bound Solute Dialysis. Colloid Surface A 2012, 405, 38-44. https://doi.org/10.1016/j.colsurfa.2012.04.032.

(19) Välimäki, S.; Khakalo, A.; Ora, A.; Johansson, L.-S.; Rojas, O. J.; Kostiainen, M. A. Effect of PEGPDMAEMA Block Copolymer Architecture on Polyelectrolyte Complex Formation with Heparin. Biomacromolecules 2016, 17 (9), 2891-2900. https://doi.org/10.1021/acs.biomac.6b00699.

(20) Yan, J.; Pan, X.; Wang, Z.; Lu, Z.; Wang, Y.; Liu, L.; Zhang, J.; Ho, C.; Bockstaller, M. R.; Matyjaszewski, K. A Fatty Acid-Inspired Tetherable Initiator for Surface-Initiated Atom Transfer Radical Polymerization. Chem. Mater. 2017, 29 (11), 4963-4969. https://doi.org/10.1021/acs.chemmater.7b01338.

(21) J. Hofer, C.; Zlateski, V.; R. Stoessel, P.; Paunescu, D.; M. Schneider, E.; N. Grass, R.; Zeltner, M.; J. Stark, W. Stable Dispersions of Azide Functionalized Ferromagnetic Metal Nanoparticles. Chem. Commun. 2015, 51 (10), 1826-1829. https://doi.org/10.1039/C4CC06126H.

(22) Zeltner, M.; N. Grass, R.; Schaetz, A.; B. Bubenhofer, S.; A. Luechinger, N.; J. Stark, W. Stable Dispersions of Ferromagnetic Carbon-Coated Metal Nanoparticles : Preparation via Surface Initiated Atom Transfer Radical Polymerization. J. Mater. Chem. 2012, 22 (24), 12064-12071. https://doi.org/10.1039/C2JM31085F.

(23) Hong, Y.; Shi, H.; Shu, X.; Zhang, Y.; Wu, Y. Tunable Synthesis of Hierarchical Superparamagnetic Fe3O4 Nanospheres by a Surfactant-Free Solvothermal Method. J. Supercond. Nov. Magn. 2018, 31 (9), 2959-2967. https://doi.org/10.1007/s10948-017-4533-3.

(24) Dong, H.; Huang, J.; Koepsel, R. R.; Ye, P.; Russell, A. J.; Matyjaszewski, K. Recyclable Antibacterial Magnetic Nanoparticles Grafted with Quaternized Poly(2-(Dimethylamino)Ethyl Methacrylate) Brushes. Biomacromolecules 2011, 12 (4), 1305-1311. https://doi.org/10.1021/bm200031v.

(25) Andrade, M. F. C.; Parussulo, A. L. A.; Netto, C. G. C. M.; Andrade, L. H.; Toma, H. E. Lipase Immobilized on Polydopamine-Coated Magnetite Nanoparticles for Biodiesel Production from Soybean Oil. Biofuel Res. J. 2016, 3 (2), 403-409. https://doi.org/10.18331/BRJ2016.3.2.5.

(26) Bonkovoski, L. C.; Martins, A. F.; Bellettini, I. C.; Garcia, F. P.; Nakamura, C. V.; Rubira, A. F.; Muniz, E. C. Polyelectrolyte Complexes of Poly[(2-Dimethylamino) Ethyl Methacrylate]/Chondroitin Sulfate Obtained at Different PHs: I. Preparation, Characterization, Cytotoxicity and Controlled Release of Chondroitin Sulfate. Int. J. Pharm. 2014, 477 (1), 197-207. https://doi.org/10.1016/j.ijpharm.2014.10.017.

(27) Vamvakaki, M.; Unali, G.-F.; Bütün, V.; Boucher, S.; Robinson, K. L.; Billingham, N. C.; Armes, S. P. Effect of Partial Quaternization on the Aqueous Solution Properties of Tertiary Amine-Based Polymeric Surfactants: Unexpected Separation of Surface Activity and Cloud Point Behavior. Macromolecules 2001, 34 (20), 6839-6841. https://doi.org/10.1021/ma010844i.

(28) Echeverria, C.; Aragón-Gutiérrez, A.; Fernández-García, M.; Muñoz-Bonilla, A.; López, D. Thermoresponsive Poly(N-Isopropylacrylamide-Co-Dimethylaminoethyl Methacrylate) Microgel Aqueous Dispersions with Potential Antimicrobial Properties. Polymers 2019, 11, 606. https://doi.org/10.3390/polym11040606.

(29) Herrmann, I. K.; Bernabei, R. E.; Urner, M.; Grass, R. N.; Beck-Schimmer, B.; Stark, W. J. Device for Continuous Extracorporeal Blood Purification Using Target-Specific Metal Nanomagnets. Nephrol. Dial. Transplant. 2011, 26 (9), 2948-2954. https://doi.org/10.1093/ndt/gfq846.

(30) Jung, S. H.; Hahn, Y. K.; Oh, S.; Kwon, S.; Um, E.; Choi, S.; Kang, J. H. Advection Flows-Enhanced Magnetic Separation for High-Throughput Bacteria Separation from Undiluted Whole Blood. Small 2018, 14 (34), 1801731. https://doi.org/10.1002/smll.201801731.

(31) Guo, L.; Zhang, L.; Zhang, J.; Zhou, J.; He, Q.; Zeng, S.; Cui, X.; Shi, J. Hollow Mesoporous Carbon Spheres-an Excellent Bilirubin Adsorbent. Chem. Commun. 2009, No. 40, 6071-6073. https://doi.org/10.1039/B911083F. 
(32) Jiang, X.; Xiang, T.; Xie, Y.; Wang, R.; Zhao, W.; Sun, S.; Zhao, C.-S. Functional Polyethersulfone Particles for the Removal of Bilirubin. J Mater Sci: Mater Med 2015, 27 (2), 28. https://doi.org/10.1007/s10856-015-5642-9.

(33) Schulman, S.; Beyth, R. J.; Kearon, C.; Levine, M. N. Hemorrhagic Complications of Anticoagulant and Thrombolytic Treatment: American College of Chest Physicians Evidence-Based Clinical Practice Guidelines (8th Edition). Chest 2008, 133 (6, Supplement), 257S-298S. https://doi.org/10.1378/chest.080674.

(34) Sokolowska, E.; Kalaska, B.; Miklosz, J.; Mogielnicki, A. The Toxicology of Heparin Reversal with Protamine: Past, Present and Future. Expert Opin. Drug Metab. Toxicol. 2016, 12 (8), 897-909. https://doi.org/10.1080/17425255.2016.1194395.

(35) Välimäki, S.; Khakalo, A.; Ora, A.; Johansson, L.-S.; Rojas, O. J.; Kostiainen, M. A. Effect of PEGPDMAEMA Block Copolymer Architecture on Polyelectrolyte Complex Formation with Heparin. Biomacromolecules 2016, 17 (9), 2891-2900. https://doi.org/10.1021/acs.biomac.6b00699.

(36) Yancheva, E.; Paneva, D.; Danchev, D.; Mespouille, L.; Dubois, P.; Manolova, N.; Rashkov, I. Polyelectrolyte Complexes Based on (Quaternized) Poly[(2-Dimethylamino)Ethyl Methacrylate]: Behavior in Contact with Blood. Macromol. Biosci. 2007, 7 (7), 940-954. https://doi.org/10.1002/mabi.200700056.

(37) Moreau, E.; Domurado, M.; Chapon, P.; Vert, M.; Domurado, D. Biocompatibility of Polycations: In Vitro Agglutination and Lysis of Red Blood Cells And In Vivo Toxicity. J. Drug Target. 2002, 10 (2), 161-173. https://doi.org/10.1080/10611860290016766.

(38) Cai, J.; Yue, Y.; Rui, D.; Zhang, Y.; Liu, S.; Wu, C. Effect of Chain Length on Cytotoxicity and Endocytosis of Cationic Polymers. Macromolecules 2011, 44 (7), 2050-2057. https://doi.org/10.1021/ma102498g.

(39) Huang, S.-J.; Ke, J.-H.; Chen, G.-J.; Wang, L.-F. One-Pot Synthesis of PDMAEMA-Bound Iron Oxide Nanoparticles for Magnetofection. J. Mater. Chem. B 2013, 1 (43), 5916-5924. https://doi.org/10.1039/C3TB21149E.

(40) Rothen-Rutishauser, B. M.; Schürch, S.; Haenni, B.; Kapp, N.; Gehr, P. Interaction of Fine Particles and Nanoparticles with Red Blood Cells Visualized with Advanced Microscopic Techniques. Environ. Sci. Technol. 2006, 40 (14), 4353-4359. https://doi.org/10.1021/es0522635.

(41) Chiu, A.; Fan, S. T. MARS in the Treatment of Liver Failure: Controversies and Evidence. Int. J. Artif. Organs 2006, 29 (7), 660-667. https://doi.org/10.1177/039139880602900703.

(42) Koivusalo, A.-M.; Vakkuri, A.; Höckerstedt, K.; Isoniemi, H. Experience of MARS Therapy With and Without Transplantation in 101 Patients With Liver Insufficiency. Transplant. Proc. 2005, 37 (8), 33153317. https://doi.org/10.1016/j.transproceed.2005.09.007.

(43) Gong, D.; Ji, D.; Zhu, D.; Xu, B.; Liu, Z. Efficient Removal of Serum Bilirubin by a Novel Artificial Liver Support System Using Albumin Convection: A Pilot Study. Blood Purif. 2012, 34 (3-4), 201-208. https://doi.org/10.1159/000342111.

(44) Rifai, K. Fractionated Plasma Separation and Adsorption: Current Practice and Future Options: FPSA: Current Practice and Future Options. Liver Int. 2011, 31, 13-15. https://doi.org/10.1111/j.14783231.2011.02595.x.

(45) Jakubowski, W. Adapting Atom Transfer Radical Polymerization to Industrial Scale Production: The Ultimate ATRPSM Technology. In Progress in Controlled Radical Polymerization: Mechanisms and Techniques; ACS Symposium Series; American Chemical Society, 2012; Vol. 1100, pp 203-216. https://doi.org/10.1021/bk-2012-1100.ch013.

(46) Keevend, K.; Panzarasa, G.; Starsich, F. H. L.; Zeltner, M.; Spyrogianni, A.; Tsolaki, E.; Fortunato, G.; Pratsinis, S. E.; Bertazzo, S.; Herrmann, I. K. Facile MeltPEGylation of Flame-Made Luminescent Tb3+-Doped Yttrium Oxide Particles: Hemocompatibility, Cellular Uptake and Comparison to Silica. Chem. Commun. 2018, 54 (23), 2914-2917. https://doi.org/10.1039/C7CC09402G.

(47) Liu, P.; Wang, Surface-initiated atom transfer radical polymerization of hydroxyethyl acrylate from activated carbon powder with homogenized surface groups. Surf. Rev. Lett. 2007, 14 (02), 269-275. https://doi.org/10.1142/S0218625X07009359. 


\section{TABLE OF CONTENTS (TOC)}

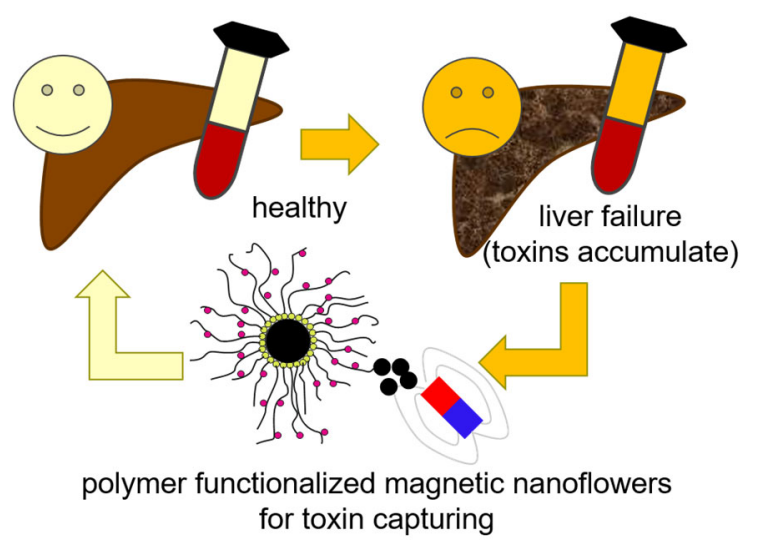

